\title{
BDD-NET - Ein internetbasiertes Programm bei Körperunzufriedenheit zur niedrigschwelligen Behandlung der körperdysmorphen Störung für den deutschen Sprachraum
}

\author{
Marie Drüge $^{\mathrm{a}} \quad$ Ulrike Buhlmann $^{\mathrm{b}} \quad$ Fanny Alexandra Dietel $^{\mathrm{b}} \quad$ Jana Hansmeier $^{\mathrm{c}}$ \\ Anne Jäger ${ }^{a} \quad$ Olenka Dworakowski $^{a} \quad$ Christian Rück $^{d}$ Jesper Enander ${ }^{d}$ \\ Birgit Watzke ${ }^{a}$ \\ ${ }^{a}$ Klinische Psychologie mit Schwerpunkt Psychotherapieforschung, Institut für Psychologie, Universität Zürich,

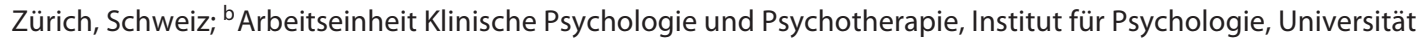 \\ Münster, Münster, Deutschland; ' AG Klinische Psychologie und Psychotherapie, Institut für Psychologie, Universität \\ Leipzig, Leipzig, Deutschland; 'Department of Clinical Neuroscience, Karolinska Institutet, Stockholm, Schweden
}

\author{
Schlüsselwörter \\ Fernbehandlung $\cdot$ Internet-Therapie $\cdot$ Kognitive \\ Verhaltenstherapie $\cdot$ Körperdysmorphe \\ Störung · Körperunzufriedenheit · Manual · \\ Zwangsspektrumsstörungen
}

\section{Zusammenfassung}

Hintergrund: Die Körperdysmorphe Störung (KDS) ist eine schwerwiegende psychische Störung, die mit starker Scham sowie Leidensdruck und Funktionseinschränkungen einhergeht. Kognitive Verhaltenstherapie stellt die aktuell wirksamste evidenzbasierte Behandlungsmethode dar. Aufgrund zahlreicher Behandlungsbarrieren kommen KDS-Betroffene jedoch selten in der psychotherapeutischen Behandlung an, weshalb niedrigschwelligen Behandlungsmethoden (z.B. aus dem Bereich E-Mental-Health) eine wichtige Rolle zukommen könnten. Erste internationale Studien weisen auf die Wirksamkeit von E-Mental-Health-Angeboten bei KDS hin. Material und Methoden: Dieser Beitrag gibt eine Übersicht zur bisherigen Umsetzung und Evidenz von E-Mental-Health-Angeboten für KDS und stellt die ins Deutsche übersetzte Version des BDD-NET-Programms, eines internetbasierten, manualisierten, therapeutenbegleiteten Interventionsprogramms, für die KDS vor, welches aus dem Englischen für den deutschen Sprachraum übersetzt und adaptiert wurde. Ergebnisse: BDD-
NET umfasst acht Module, die binnen einer 12-wöchigen Behandlung online bearbeitet werden. Die Online-Plattform bietet für die Patienten die Möglichkeit, mit dem BDD-NETTherapeuten mittels persönlicher Nachrichten zu kommunizieren. Sämtliche Materialien wurden aus dem Englischen übersetzt und vor allem in kultureller Hinsicht adaptiert. Schlussfolgerungen: BDD-NET könnte ein wichtiger Baustein in der Versorgung von KDS-Betroffenen sein. Die Evaluation steht für den deutschen Sprachraum noch aus. Auf Besonderheiten des Settings (z.B. Störungseinsicht als möglicher Behandlungsfokus) sowie praktische Implikationen wird eingegangen. Zudem werden Voraussetzungen und Rahmenbedingungen für eine perspektivische Dissemination diskutiert.

(c) 2021 The Author(s)

Published by S. Karger AG, Basel

BDD-NET - An Internet-Based Program as a LowThreshold Treatment for Body Dysmorphic Disorder: A German Treatment Program

\section{Keywords}

Remote treatment · Internet interventions · Cognitive behavioral therapy - Body dysmorphic disorder . Body dissatisfaction - Manual - Obsessive compulsive spectrum disorders mercial purposes requires written permission. 


\begin{abstract}
Background: Body dysmorphic disorder (BDD) is a mental disorder that is associated with pronounced shame, a high burden of disease, and functional impairments. Cognitive behavioral therapy (CBT) has been found the most effective treatment for BDD. However, due to several treatment barriers, most individuals with BDD do not receive adequate psychotherapeutic treatment, which is why low-threshold treatment options (e.g., E-Mental Health) could play an important role to fill this gap. Prior international studies have demonstrated the effectiveness of E-Mental Health interventions for BDD. Material and Methods: This article provides an overview of the literature regarding the implementation and effectiveness of E-Mental Health interventions for BDD and introduces the German version of BDD-NET, a manualized, internetadministered cognitive-behavioral program for BDD. $\boldsymbol{R e}$ sults: BDD-NET consists of eight online modules delivered over 12 weeks. Further, the online platform provides the possibility to contact the therapist. In the current German version, all materials were translated from the original, and adaptations were made with regard to cultural aspects. Conclusions: BDD-NET could constitute an important component in specific care options for BDD. To date, evaluation of the German version is still pending. Special characteristics of the online setting as well as clinical implications (e.g., insight as a potential treatment target) are presented. Conditions and structural factors for prospective dissemination are discussed.

(c) 2021 The Author(s).

Published by S. Karger AG, Basel
\end{abstract}

\section{Einleitung und Überblick}

Obwohl es in den letzten Jahren ein zunehmendes Interesse an der Körperdysmorphen Störung (KDS) in Forschung und Praxis gibt, besteht ein hoher Forschungsbedarf sowie ein Bedarf an der Weiterentwicklung und Ausweitung des Behandlungsangebotes für Betroffene. Der vorliegende Beitrag stellt die deutsche Übersetzung des in Schweden entwickelten und evaluierten, internetgestützten Behandlungsprogramms BDD-NET [Enander at al., 2014, 2016] vor. Im Folgenden werden hierzu das klinische Bild der KDS sowie therapierelevante Besonderheiten und Behandlungsmöglichkeiten (u.a. E-MentalHealth-Angebote) dargestellt. Schließlich wird die deutsche Version von BDD-NET als internetgestützte Behandlungsoption inklusive der aktuell laufenden Effektivitätsstudie präsentiert.

\section{Hintergrund}

\section{Die Körperdysmorphe Störung}

Bei der KDS manifestiert sich ein ausgeprägter Leidensdruck und/oder eine Beeinträchtigung durch die übermäßige Beschäftigung mit einem oder mehreren wahrgenommenen Makeln bzw. Merkmalen des äußeren Erscheinungsbildes (z.B. unregelmäßiges Hautbild, Nasenform, kleine Brüste), die von Dritten nicht oder nur geringfügig erkennbar sind. Die exzessive Aussehensbeschäftigung führt typischerweise $\mathrm{zu}$ ausgeprägtem ritualisierten Sicherheits- und Vermeidungsverhalten [American Psychiatric Association, 2013]. Im ICD10 noch den Somatoformen Störungen bzw. der hypochondrischen Störung zugeordnet, ist die KDS im ICD-11, welches künftig in Kraft treten wird, in der Gruppe der Zwangs- und Zwangsspektrumsstörungen als eigenständige Diagnose zu finden [World Health Organization, 2019, 2020a]. Insgesamt wird die Punktprävalenz auf 1,9\% geschätzt [Buhlmann et al., 2010; Veale et al., 2016]. In 70\% der Fälle liegt der Störungsbeginn bereits in der Adoleszenz, im Durchschnitt mit 16 Jahren [Gunstad und Phillips, 2003]. Laut einer Untersuchung von Gunstad und Phillips [2003] ( $N=293)$ geht die KDS bei 75,5\% der Betroffenen komorbid mit einer Major Depression einher, bei 36,5\% mit einer Sozialen Angststörung und bei 32,1\% mit einer Zwangsstörung. Des Weiteren waren komorbide substanzinduzierte Störungen (Alkohol: 20,5\%; andere Substanzen: 17,1\%) häufig.

Die KDS ist im Rahmen veränderter Informationsverarbeitungsprozesse (z.B. negativer Aufmerksamkeitsund Interpretationsverzerrungen, die in selbstabwertenden Kognitionen münden) mit vielen negativen Emotionen - insbesondere Scham - verbunden [Wilhelm et al., 2013]. Diese Emotionen führen verstärkt zu kompensatorischen behavioralen (z.B. exzessive Körperpflege, kaschierende Bekleidung, plastisch-chirurgische Eingriffe) oder mentalen Handlungen (z.B. sich aufdrängender, fortdauernder Vergleich mit dem Aussehen anderer) mit ritualisiertem Charakter. Ritualisierte Handlungen kosten die Betroffenen viel Zeit und Energie, ca. 40\% der Personen mit KDS verbringen drei bis acht Stunden und 25\% mehr als acht Stunden täglich mit aussehensbezogenen Ritualen, Vermeidungs- und Sicherheitsverhalten [Phillips, 2005].

Im Verlauf der Störung leiden viele Betroffene an psychosozialen Einschränkungen und damit einhergehender geringer Lebensqualität bzw. funktionaler Gesundheit [Phillips et al., 2005; Schneider et al., 2019]. Dies schlägt sich im Meiden von Sozialkontakten und (intimen) Beziehungen nieder und kann bis hin zur völligen Isolation sowie zur Erwerbsunfähigkeit führen [Kelly et al., 2017]. In einer Studie von Didie et al. [2008] waren 
39\% der 141 KDS-Betroffenen im vergangenen Monat völlig arbeitsunfähig. Zudem ist bei der KDS eine hohe Suizidalitätsrate zu verzeichnen. In einer prospektiven Studie über vier Jahre berichteten pro Jahr 57,8\% der 185 Betroffenen Suizidgedanken, 2,6\% der Betroffenen begingen einen Suizidversuch, und 0,3\% nahmen sich pro Jahr das Leben [Phillips und Menard, 2006].

Fehldiagnosen sind eine häufige Komplikation innerhalb der klinischen Praxis der KDS-Behandlung [Buhlmann et al., 2011]. In einer neueren Untersuchung wurden lediglich 15,2\% der 429 KDS-Betroffenen entsprechend diagnostiziert [Schulte et al., 2020]. Eine falsche oder fehlende Diagnose kann zudem einen der Erklärungsfaktoren bilden, warum lediglich 17,4\% der Betroffenen eine kognitive Verhaltenstherapie (KVT) erhält [Marques et al., 2011]. Betroffene fallen eher aufgrund von psychischen Komorbiditäten und (Sekundär-)Problematiken, wie beispielsweise einer depressiven Symptomatik, auf. Die fehlende oder zeitverzögerte Initiierung von Fachbehandlungen ist umso kritischer einzuschätzen, als dass die KDS unbehandelt typischerweise chronisch verläuft [Phillips et al., 2013].

\section{Behandlungsmöglichkeiten der KDS}

First-line Behandlungen sind als wirksamste Therapiemöglichkeiten nach aktuellem Forschungsstand die KVT [Veale und Neziroglu, 2010; Wilhelm et al., 2013] und die medikamentöse Behandlung mit selektiven Serotonin-Wiederaufnahmehemmern (SSRIs, z.B. Fluoxetin) [Castle et al., 2021]. Die einzigen klinischen Leitlinien, welche Empfehlungen zur Behandlung der KDS beinhalten, stammen vom National Institute for Health and Care Excellence [NICE, 2005]. Sie führen die Empfehlungen zur Behandlung der KDS zusammen mit denen für Zwangsstörungen auf. Empfohlen wird je nach Schweregrad der Symptomatik sowohl für Kinder, junge Erwachsene und Erwachsene eine psychotherapeutische Behandlung, vorzugsweise eine KVT, und die psychopharmakologische Behandlung mit SSRIs (Fluoxetin, Fluvoxamin, Paroxetin, Sertralin oder Citalopram) als Kombinationsbehandlung. Die Einnahme des SSRI wird für mindestens 12 Wochen empfohlen; zeigt sich das SSRI hierbei effektiv, sollte es folgend für 12 Monate eingenommen werden, um Rückfälle zu vermeiden [NICE, 2005]. Für die Wirksamkeit von KVT zur Behandlung einer KDS gibt es mittlerweile erste vielversprechende Ergebnisse. Die jüngste Metaanalyse zur Wirksamkeit von KVT bei KDS mit sieben randomisiert-kontrollierten Studien konnte zeigen, dass die Reduktion der KDS-Symptomatik der Interventionsgruppen signifikant grösser war als in den Kontrollgruppen $(d=-1,2)$, die in den Vergleichsbedingungen variierten (z.B. Wartelistekontrolldesign oder supportive Therapie) [Harrison et al., 2016]. Drei der in die Metaanalyse eingeschlossenen Studien umfassten hierbei FollowUp-Erhebungen. Diese deuteten darauf hin, dass die Effekte auch noch innerhalb von zwei bis vier Monaten nach Behandlungsabschluss vorhanden waren. Hierbei ist jedoch zu beachten, dass das Setting der in die Metaanalyse eingeschlossenen KVT-Behandlungen variierte: Von der Gesamtstichprobe der 299 Betroffenen erhielten die 94 Personen eine internetgestützte Therapie und weitere 54 Personen eine Gruppentherapie. Die übrigen 148 Betroffenen verteilen sich auf fünf randomisiert kontrollierten Studien (RCT) mit folglich kleinen Fallzahlen. Für die psychopharmakologische Behandlung der KDS erwiesen sich SSRIs als wirksam [Castle et al., 2021]. Sie konnten den Schweregrad der KDS-Symptomatik und die damit verbundene Belastung senken. Castle et al. [2021] gehen neben der längeren Gabe von SSRIs zudem auf weitere adjunktive Therapien (z.B. intranasal appliziertes Oxytocin) ein. Insgesamt zeigt sich, dass die Datengrundlage zwar vielversprechend, gleichzeitig jedoch noch nicht zuverlässig ist. Es bedarf weiterer RCTs, z.B. mit höheren Fallzahlen und einer stärkeren Berücksichtigung von Komorbiditäten, um eine zuverlässige Datengrundlage zu bieten und die externe Validität zu sichern.

\section{E-Mental-Health und KDS}

Sowohl das Angebot als auch die Nachfrage von EMental-Health-Angeboten ist in den letzten Jahren gestiegen. Die COVID-19-Pandemie hat diese Nachfrage nochmals stimuliert [Wong et al., 2021] und u.a. zu einem massiven Innovationsschub bei Videobehandlungen geführt [Bundespsychotherapeutenkammer, 2020]. Zudem verlängerten sich zum Teil Wartezeiten für eine Psychotherapie oder es kam während des Lockdowns zu Unterbrechungen der Psychotherapie [Boldrini et al., 2020]; in $70 \%$ der Länder wurden COVID-19-bedingte Unterbrechungen des Face-to-Face Kontaktes durch telemedizinische Optionen (Psychotherapie via Videokonferenz oder Telefon) ersetzt [World Health Organization, 2020b]. Im Zusammenhang mit E-Mental-Health-Interventionen werden einerseits Vorteile - etwa die höhere Erreichbarkeit, Reichweite und Aktualität - als auch Nachteile - beispielsweise fraglicher Datenschutz oder Schwierigkeiten im Umgang mit Krisensituationen - vielfach diskutiert [Moessner und Bauer, 2017].

Allerdings bieten E-Mental-Health-Angebote insbesondere für ansonsten schwierig erreichbare Patientengruppen (z.B. KDS-Betroffene, die das Haus nicht verlassen können) aufgrund ihrer Niedrigschwelligkeit eine zusätzliche Option, den Zugang zu einer (ersten) Behandlung zu erleichtern. Dementsprechend wurden, ergänzend zum Face-to-Face-Angebot, mittlerweile inter- 
netgestützte Ansätze als psychologische Unterstützungsangebote zur Selbsthilfe (guided self-help) auch für KDS entwickelt, deren Effektivität in ersten Studien gezeigt werden konnte. Neben der Abnahme der KDS-Symptomatik sind zudem auch Patienteneinschluss, DropoutQuote sowie Masse der Feasibility (z.B. Akzeptanz und Zufriedenheit) sowie Gründe zur Teilnahme interessant, um den Nutzen von Internet-gestützten Behandlungen $\mathrm{zu}$ beschreiben. In schwedischer sowie in englischer Sprache steht das internetgestützte Programm BDDNET zur Verfügung, welches zum jetzigen Zeitpunkt das am besten evaluierte internetgestützte Programm für KDS darstellt [Enander et al., 2014, 2016, 2019; Gentile et al., 2019]. Eine Studienreihe (Pilotierung + RCT + Follow-Up) untersuchte BDD-NET auf Schwedisch, eine weitere internationale Studie in der englischen Sprache. In einer ersten Studie zur Feasibility [Enander et al., 2014] zeigte sich bei den 23 teilnehmenden KDS-Betroffenen eine hohe Akzeptanz und Zufriedenheit mit BDDNET: $85 \%$ waren (sehr) zufrieden mit dem Programm. Zudem wurden signifikante Verbesserungen in der BDD-YBOCS ( $p \leq 0,001$, within-group Effektstärke Cohen's $d=2,01$, KI 95\%, 1,05-2,97) berichtet, und 82\% der Teilnehmenden als Responder $(\geq 30 \%$ Verbesserung in der BDD-YBOCS) definiert. Diese Ergebnisse wurden in einer single-blind randomisiert kontrollierten Studie mit 94 KDS-Betroffenen mit einer supportiven Therapie verglichen. BDD-NET zeigte sich der supportiven Therapie als überlegen (BDD-YBOCS Zwischengruppeneffekte: $-7,1$ Punkte, KI 95\%, $-9,8$ bis $-4,4$ Punkte, $d=0,87$ drei Monate nach Programmende) [Enander et al., 2016]. Während sich in der Gruppe der supportiven Therapie nach 3-monatigem Follow-Up nur 16\% Responder ( $\geq 30 \%$ Verbesserung im BDD-YBOCS) fanden, zeigten sich in der Gruppe von BDD-NET 56\% Responder. Im 1-Jahres-Follow-Up $(N=54)$ zeigte sich im Vergleich zur Baseline eine signifikante Verbesserung des BDDYBOCS ( $p \leq 0,001$, within-group Effekte -12,3 Punkte; KI 95\% -14,53 bis $-10,25$ Punkte, $d=1,72$ ) [Enander et al., 2019]. Auch im 2-Jahres-Follow-Up $(N=65)$ zeigten sich 69\% unter den oben genannten Kriterien als Responder [Enander et al., 2019]. Im Vergleich zur Baseline zeigte sich eine signifikante Verbesserung des BDDYBOCS ( $p \leq 0,001$, within-group Effekte -13,42 Punkte; KI 95\% $-15,57$ bis $-11,27$ Punkte, $d=1,79$ ). Insgesamt erfüllten zum Zeitpunkt des Langzeit-Follow-Ups gemäß eines strukturierten klinischen Interviews 56\% (KI 95\% 43\% bis 69\%) die DSM-5 Kriterien einer KDS nicht mehr. Die Wirksamkeit von BDD-NET erwies sich folglich auch über zwei Jahre als stabil, wobei diese unter Einbezug der Selektivität des Dropouts zum Follow-Up (56 von ursprünglich 94 randomisierten Patienten) interpretiert werden sollte.
In einer unkontrollierten Studie untersuchten Gentile et al. [2019] BDD-NET an 32 KDS-Betroffenen aus neun verschiedenen englischsprachigen Ländern. Es zeigte sich eine signifikante Verbesserung der BDD-YBOCS Mittelwerte von Baseline zu Woche $6(-8,12)$, zum Therapieende $(-12,63)$ und zum 3-Monats-Follow-Up $(-11,71) .50 \%$ der Teilnehmenden wurden beim FollowUp als Responder definiert, allerdings beendeten nur 25 Personen die Behandlung. Die Remissionsrate lag nach der Behandlung bei $28 \%$ und beim Follow-Up bei $44 \%$. Dieser - im Vergleich zur Hauptstudie der schwedischen Gruppe - hohe Dropout der Studie von Gentile et al. [2019] nähert sich dem von anderen internetgestützten Interventionen an, könnte allerdings auch durch den globalen Einschluss (weniger Eingehen auf kulturelle Unterschiede möglich) erklärt werden. Bemerkenswert ist der hohe Patienteneinschluss in der Hauptstudie [Enander et al., 2016] trotz krankheitsimmanenter hoher Scham, der möglicherweise das Internet als gute $\mathrm{Zu}$ gangsmöglichkeit - zumindest für eine Subgruppe der KDS-Betroffenen - positioniert. Dies wurde auch in einem Interview zu Gründen zur ursprünglichen Teilnahme zum 1-Jahres-Follow-Up deutlich [Enander et al., 2019]. Von den 49 Teilnehmenden gaben $63 \%$ an, dass sie sich ohne die Studie keine Hilfe gesucht hätten. Für 63\% war der Hauptgrund zur Teilnahme die Möglichkeit einer Behandlung, ohne zur Klinik oder Praxis fahren zu müssen. $44 \%$ gaben an, dass es für sie möglich war teilzunehmen, da sie nicht Face-to-Face in einer therapeutischen Sitzung sitzen könnten (z.B. aufgrund von Scham) [Enander et al., 2019]. Umso überraschender erscheint der - vergleichsweise - geringe Patienteneinschluss in der internationalen Studie [Gentile et al., 2019], für die auf unterschiedlichen Wegen (z.B. ärztliche Überweisung, Google, soziale Medien) rekrutiert wurde und der möglicherweise auf Schwierigkeiten in der Akzeptanz neuartiger Therapieangebote oder den wichtigen lokalen Bezug hindeuten könnte.

Wilhelm et al. [2020] haben zudem das erste Smartphone-gestützte Programm für KDS entwickelt, das eine 12-wöchige KVT beinhaltet. In einer ersten Pilotierung zeigte sich eine positive Wirkung der Intervention; in dem Fremdbeurteilungsinstrument zur Einschätzung der KDS-Symptomatik, der BDD-YBOCS [Phillips et al., 1997], nahm die KDS-Symptomatik über die Therapie ab $(\mathrm{M}=45,27 \%, \mathrm{SD}=14,66 \%, d=2,60)$. Neun der Teilnehmer (90\%) wurden gemäß dem Kriterium ( $\geq 30 \%$ Verbesserung in der BDD-YBOCS) nach der Therapie als Responder definiert, was sich auch im 3-Monats-Follow-Up stabil zeigte. In einer aktuellen zweiarmigen RCT wird derzeit ImaginYouth [Hartmann et al., 2021], eine weitere 12-wöchige, internetgestützte, KDS-spezifische KVT, gegen eine supportive, internetgestützte Intervention getestet.
Drüge et al. 


\section{Das computergestützte Programm für KDS “BDD-NET - Ein internetbasiertes Programm bei Körperunzufriedenheit"}

BDD-NET wurde in Schweden am Karolinska-Institutet als internetbasiertes kognitiv-verhaltenstherapeutisches Interventionsprogramm für KDS-Betroffene entwickelt und ausführlich evaluiert [Enander et al., 2014, 2016, 2019; Gentile et al., 2019].

Bisher liegt das Programm ausschließlich in englischer und schwedischer Sprache vor. Im Folgenden wird zunächst die Intervention beschrieben, bevor die Übersetzung mit kulturellen sowie leichten inhaltlichen Adaptionen ins Deutsche vorgestellt sowie kurz auf die wissenschaftliche Evaluation der deutschsprachigen Version eingegangen wird.

\section{Interventionsbeschreibung}

BDD-NET ist als kognitiv-verhaltenstherapeutische Kurzzeitintervention über einen Zeitraum von 12 Wochen konzipiert und basiert auf dem modularisierten Behandlungsansatz nach Wilhelm et al. [2013]. Es soll Patienten dabei helfen, Symptome der KDS selbstständig zu erkennen und diese adaptiv zu bewältigen, indem es direkt an störungsaufrechterhaltenden Faktoren (u.a. negativ verzerrten Kognitionen, Vermeidungsverhalten) durch spezifische Interventionen (u.a. kognitive Umstrukturierung, Expositionen) ansetzt. BDD-NET ist ein internetgestütztes Programm, in das sich Patient sowie BDD-NET-Therapeut gemeinsam über eine desktopbasierte Plattform einloggen können. Dem Patienten werden hierbei sukzessive Module zur selbstständigen Bearbeitung dargeboten, die Instruktionstexte, Übungen und Hausaufgaben umfassen. Die Module werden eigenständig durch den Patienten erarbeitet, Übungen können online ausgefüllt, abgegeben und somit an den Therapeuten übermittelt werden. Weiterhin hat der Patient über das Programm die Möglichkeit, seinem Therapeuten Nachrichten zu schreiben. Die Online-Plattform ist nach geltenden, regelmäßig aktualisierten Verschlüsselungsstandards implementiert und enthält erweiterte Sicherungsmaßnahmen (u.a. Zwei-Faktor-Authentifizierung bei Therapeuten-Login). Alle Materialien sind direkt auf der Plattform verfügbar und prinzipiell auch in PDF- oder Papierform konvertierbar.

Die ersten Module sollen in der Regel wöchentlich bearbeitet werden, die Bearbeitungsdauer beträgt pro Modul ca. 60 Minuten. Allein die übungsintensiveren Expositionsmodule umfassen eine Bearbeitungszeit von 4 Wochen. Alltagsnahe Übungen werden durch Arbeitsblätter angeregt, um das Üben zwischen den Modulen zu fördern. Die Übung "KDS-Tagebuch" wird beispielsweise in Modul 1 eingeführt und soll dabei helfen, Symptome der KDS (z.B. Vermeidens-/Sicherheitsverhalten) wahrzu- nehmen sowie die Symptome als veränderbar einzuordnen. Jedes Modul (außer Modul 1) beginnt mit einem Rückblick zum letzten Modul. Hierauf folgt die Zielsetzung des folgenden Moduls. Den Abschluss bilden reflektierende Wissensfragen.

Das Programm umfasst acht Module, welche etablierte Elemente der kognitiv-verhaltenstherapeutischen Behandlung der KDS (z.B. Psychoedukation, kognitive Umstrukturierung, Exposition und Reaktionsverhinderung, Werteorientierung, Rückfallprophylaxe) beinhalten. Der inhaltliche Ablauf deckt sich mit jenem kognitiv-verhaltenstherapeutischer Behandlungen der KDS [Veale und Neziroglu, 2010; Brunhoeber, 2019; Hartmann et al., 2019] und orientiert sich dabei stark an dem Behandlungsmanual von Wilhelm et al. [2013], welches den Schwerpunkt stärker auf den behavioralen als auf den kognitiven Behandlungsaspekt legt.

- Modul 1 "Fragen und Antworten zur Körperdysmorphen Störung" umfasst Psychoedukation über die Symptome der KDS, welche durch eine Fallvignette illustriert werden. Die Begrifflichkeiten des Körperbilds sowie der Körperwahrnehmung werden erklärt, zudem werden die Prävalenz sowie biopsychosoziale Ursachen für eine KDS erklärt. Sicherheits- und Vermeidungsverhalten werden begrifflich eingeführt. Der Patient wird zur eigenen Reflexion ermuntert.

- Modul 2 "Ein psychologisches Erklärungsmodell der KDS" dient ebenfalls der Psychoedukation. Es erläutert die wechselseitige Dreiecksbeziehung zwischen Gedanken, Gefühlen und Verhalten. Zudem wird ein Teufelskreismodell dargestellt, indem aufgegriffen wird, warum sich jemand wegen seines Aussehens unter anderem ängstlich oder angewidert fühlen kann und welche Faktoren dazu führen, dass diese belastenden Gefühle bestehen bleiben. Besonders wird auf verschiedene Arten des Sicherheitsverhaltens und des Vermeidungsverhaltens eingegangen, die belastende Gefühle verringern sollen, diese jedoch tatsächlich nur kurzfristig reduzieren und auf lange Sicht KDS-Symptome verschlechtern. Zudem wird auf die KDS-immanente Schwierigkeit der Einsicht eingegangen und dadurch aufgegriffen, dass der Patient einerseits Theorie A (es liegt an dem Aussehen selber) mit Theorie B (es liegt an dem eigenen Empfinden über das Aussehen) vergleicht, um so die psychologische Ursache der Symptome zu betonen.

- Modul 3 "Gedanken und Interpretationsfallen" basiert auf der kognitiven Umstrukturierung. Mit der Zitronenübung wird der Zusammenhang von Gedanken und physiologischen Reaktionen illustriert. Anschließend werden gängige kognitive Fehler/Interpretationsfallen (z.B. Alles-oder-Nichts-Denken, Etikettieren) dargestellt und ein konstruktiver Umgang mit ihnen erläutert. Ungünstige Gedanken können so er- 
kannt und hinterfragt werden, sodass sie als weniger handlungsleitend empfunden werden können.

- Modul 4 "Einführung zur Exposition mit Reaktionsverhinderung" und Modul 5 "Mehr über Expositionen" beschäftigen sich mit der Erklärung sowie der Planung von Expositionen und Reaktionsverhinderung. Zunächst werden noch einmal Vermeidungs- und Sicherheitsverhalten, inklusive ihrer Funktionalität in Bezug auf kurz- und langfristige Folgen, aufgegriffen sowie die Verbindung zur reduzierten Lebensqualität erläutert. Über emotionale Ziele, Verhaltensziele und langfristige Ziele wird mit dem Patienten eine Motivation zur Expositionsbehandlung aufgebaut. Das anschließende Arbeitsblatt "Exposition und Reaktionsverhinderung" wird erläutert und ermöglicht das differenzierte Planen einer Exposition. In Modul 5 werden typische Szenarien während des Durchführens einer Exposition dargestellt und zum adaptiven Umgang damit motiviert, die zu einer gelingenden Expositionsbehandlung verhelfen können. Ein reflektierendes Arbeitsblatt, das äquivalente Bausteine zu dem Arbeitsblatt aus Modul 4 umfasst, soll dazu dienen, den eigenen Lernfortschritt zu illustrieren.

- Modul 6 "Wertorientierte Richtungen und Ziele" dient der Werteorientierung, um an der Lebensqualität und -zufriedenheit der Patienten anzusetzen. Es unterstützt dabei, über eigene Werte in Bezug auf Beziehungen, Arbeit und Ausbildung sowie Freizeit und Interessen $\mathrm{zu}$ reflektieren und das alltägliche Leben durch kleine Ziele in Richtung der eigenen Werte zu lenken. Viele Betroffene konzentrieren sich sehr darauf, ihr Aussehen, Gedanken und Gefühle zu kontrollieren. Dieses Modul hat zum Ziel (a) zu erkennen, was im Leben wichtig ist, und (b) damit anzufangen, im Einklang mit persönlichen Werten zu handeln, trotz störender, ungünstiger Gedanken und Gefühle. Expositionen werden weiterhin geplant und durchgeführt.

- Modul 7 "Schwierigkeiten und Rückschläge (während des Behandlungsprogramms)" dient der Rückfallprophylaxe. Übliche Schwierigkeiten und Probleme, gerade auch mit fortlaufenden Expositionen möglicherweise steigender Schwierigkeit, werden exemplarisch dargestellt (z.B. Motivationsfallen) und Lösungsempfehlungen gegeben.

- Modul 8 "Zusammenfassung und Ausblick" beschäftigt sich schließlich mit der Entwicklung eines individuellen Notfallplans, sodass jeder Patient sein eigenes Programm zur Rückfallprophylaxe entwickelt.

Auf der Online-Plattform haben die Patienten jederzeit die Möglichkeit, ihren BDD-NET-Therapeuten per Nachricht zu kontaktieren. Für den therapeutischen Kontakt (z.B. Beantworten der Nachrichten sowie Planen von Expositionen) werden über den Behandlungsverlauf 120 Minuten der therapeutischen Interaktion insgesamt eingeplant. Besonders Expositionsübungen können so abgestimmt und kleine Schritte reflektiert werden. Patienten erhalten an Wochentagen innerhalb von 48 Stunden eine Antwort des Therapeuten. Die Patienten können in der Plattform ein Jahr nach Behandlungsende auf sämtliche Materialien und Übungen zugreifen.

\section{Übersetzung mit kulturellen und inhaltlichen Adaptionen des Interventionsprogramms “BDD- NET - Ein internetbasiertes Programm bei Körperunzufriedenheit"}

Das Material besteht aus den einzelnen Modulen, Arbeitsblättern sowie Fallbeispielen und Musteraufgaben, welche durch deutsche Muttersprachlerinnen mit Fachexpertise in der Behandlung der KDS (M.D., J.H., U.B., B.W.) zunächst übersetzt wurden. Ansatzpunkte für die Weiterentwicklung des Programmes ergaben sich durch die Bearbeitungsquote, welche für einige Programmmodule relativ gering ausfiel. So wurde etwa in der Untersuchung von Enander et al. [2016] das zentrale Behandlungsmodul der Exposition nur von $61 \%$ der Teilnehmenden bearbeitet. Möglicherweise könnte dies mit der Länge des Textes bzw. Redundanzen sowie fehlender therapeutischer Unterstützung bei der Durchführung der Expositionen zusammenhängen, weshalb Überarbeitungen auf sprachlicher Ebene (z.B. Textkürzungen) sowie auf inhaltlicher Ebene (z.B. Ergänzung motivierender Abschnitte) vorgenommen wurden. Die entstandene Adaptation wurde mit dem Original abgeglichen, im Konsensverfahren in der Arbeitsgruppe (M.D., J.H., U.B., B.W.) diskutiert sowie dokumentiert. Eine bilinguale Muttersprachlerin (O.D.) wurde für sprachliche Feinheiten sowie einem abschließenden Check hinzugezogen. Änderungen und Abweichungen fanden sich auf Ebene des Wortlauts gemäß dem üblichen Fachjargon (z.B. "negative thoughts" wurde durch "ungünstige Gedanken," "negative emotions" durch "unangenehme Gefühle," "harder expositions" mit "als schwieriger bewertete Expositionen" ersetzt) und führten nicht zu relevanten inhaltlichen Unterschieden. Auf den Begriff "Hausaufgaben" wurde als direkte Übersetzung verzichtet; dieser wurde durch "Übungen" ersetzt. Gemäß den soziokulturellen Unterschieden zwischen der bisherigen Zielgruppe in englischer Sprache und dem deutschen Sprachraum wurden zahlreiche Adaptionen vorgenommen: Sämtliche Textstellen wurden beispielsweise von der Ansprache "you" in die formellere Ansprache "Sie" umformuliert. Redewendungen wie "it's better to be safe than sorry" wurden inhaltlich aufgegriffen. Ebenso wurde das im Englischen verwendete Bild der wiederkäuenden Kuh, um Rumination zu erläutern, im Deutschen durch das Gedankenkarussell ersetzt, da das Bild der Kuh eher zu 
Selbstabwertungen einlädt. Sämtliche Vornamen der Fallbeispiele wurden adaptiert und der deutsche Vorname "Maria" verwendet. Zudem wurden Passagen umstrukturiert (z.B. Erweiterung oder Verschiebung) und Redundanzen gekürzt. Motivierende Abschnitte wurden kulturell angepasst, zum Beispiel wurde "Good work!" zu "Gut gemacht!"

\section{Umsetzung des internetgestützten Therapieprogrammes}

In England wurde BDD-NET bereits in das Programm "Improving Access to Psychological Therapies" (IAPT) des National Health Services aufgenommen und wird dort zur Behandlung von Betroffenen einer mittleren bis schweren KDS - bestenfalls als Element eines steppedcare-Modells - empfohlen [NICE, 2019]. Die Kosten werden pro Durchführung auf 120 GBP geschätzt und liegen somit deutlich unter den Kosten anderer ambulanter oder stationärer, nationaler Therapieangebote (z.B. Face-to-Face [NICE, 2019]). Es kann durch geschulte Therapeuten angewandt werden. BDD-NET nutzt das "Patient Health Questionnaire 9" (PHQ-9) [deutsche Version, Kroenke und Spitzer, 2002] und das "appearance anxiety inventory" (AAI) [Veale et al., 2014] als wöchentliches Monitoring, um den jeweiligen BDD-NETTherapeuten den Fortschritt der Patienten zurückzumelden und durch Veränderungsgraphen zu verbildlichen.

\section{Wissenschaftliche Evaluation der deutschsprachigen Version}

Die Wirksamkeit (efficacy) der deutschsprachigen Version von BDD-NET wird aktuell im Rahmen eines einfach verblindeten, wartelistenkontrollierten RCT unter der Leitung beteiligter Autorinnen (U.B., F.D.) geprüft ${ }^{1}$. Hierzu werden Teilnehmende, welche die Primärdiagnose einer KDS nach DSM-5 erfüllen, deutschlandweit über verschiedene Quellen (u.a. Webpräsenz, Ambulanzen, Praxen und Kliniken sowie soziale Medien) rekrutiert. Studienteilnehmende werden zufällig der aktiven Behandlungsbedingung mit BDD-NET oder einer zeitäquivalenten Wartebedingung zugeteilt. Im ersten Fall durchlaufen Teilnehmende direkt das Behandlungsprogramm, im zweiten Fall haben Teilnehmende nach Ablauf der zwölfwöchigen Warteperiode die Möglichkeit, in das Behandlungsprogramm zu wechseln.

Spezifisch geschulte Diagnostikerinnen führen die standardisierte Initial- und Verlaufsdiagnostik via einer gesicherten Videoplattform mit den Patienten durch.

\footnotetext{
Die Studie ist im Deutschen Register Klinischer Studien (DRKS) unter der Identifikationsnummer DRKS00021726 präregistriert. Detaillierte Studieninformationen sind dort ersichtlich.
}

Hierbei kommen zentrale Klassifikations- und Symptomschweremaße (u.a. BDD-Y-BOCS [Phillips et al., 1997; deutsche Version, Stangier et al., 2000], Brown Assessment of Beliefs Scale [BABS; deutsche Version, Buhlmann, 2014] zur Messung der Störungseinsicht) zum Einsatz. Ergänzend werden Selbstauskunftsmaße, die neben der Symptomatik auch bisher nicht untersuchte, therapeutisch relevante Variablen (u.a. wahrgenommene Qualität der therapeutischen Allianz, Behandlungserwartung und -zufriedenheit) quantifizieren, dargeboten. Eine diagnostische Beurteilung erfolgt in diesem Rahmen zur Baseline, zum Mid-Treatment nach 6 Wochen (hierbei nur in Selbstauskunft), zur Post-Erhebung und zum vierwöchigen Follow-Up.

Stimmung und Suizidalität werden während der aktiven Behandlung mit BDD-NET wöchentlich geprüft. Der Überwachung der Sicherheit der Patienten kommt angesichts der hohen Suizidalitätsraten hierbei eine besondere Bedeutung zu. Innerhalb wöchentlichen Stimmungsmonitorings mittels des PHQ-9 [Kroenke und Spitzer, 2002] wird die aktuelle Suizidalität geprüft. Bei erhöhten Werten wird der Therapeut hierbei automatisch über die Plattform benachrichtigt. Für kritische Situationen (z.B. akute Suizidalität) stehen gestufte Notfallpläne mit spezifisch definierten Zuständigkeiten zur Verfügung, die eine Kontaktaufnahme (z.B. per Telefon) und bedarfsgerechte Zuweisung an intensivierte, lokale Behandlungsangebote vorsehen.

Aktuell läuft der weitere Teilnehmendeneinschluss für die erste deutschsprachige Evaluation von BDD-NET. Der Studienabschluss ist für 2022 geplant.

\section{Diskussion und Ausblick}

Es ergeben sich zahlreiche Unterschiede zwischen dem internetgestützten Programm BDD-NET und Faceto-Face-Therapien, die oftmals auf modalitätsbedingte Diskrepanzen zurückzuführen sind, wie z.B. ihre kurze Dauer, starke Strukturierung und stärkere Unverbindlichkeit. Dies führt zu den angesprochenen Vorteilen von E-Mental-Health-Angeboten, wie die höhere Skalierbarkeit, Erreichbarkeit, Reichweite und Aktualität. Demgegenüber stehen Nachteile, wie z.B. der fragliche Datenschutz oder Schwierigkeiten mit dem Umgang mit Krisensituationen [Moessner und Bauer, 2017]. Die bestmögliche Reduktion dieser Limitationen wurde in der deutschsprachigen Version von BDD-NET durch die implementierten Datensicherungsmaßnahmen und gestufte Notfallkonzepte im Besonderen berücksichtigt. Neben den generellen Vor- und Nachteilen ergeben sich auch KDS-spezifische Besonderheiten: Die Behandlungsintensität der Intervention ist als niedrigschwellig und vergleichsweise kurz zu bewerten. Die therapeuti- 
sche Interaktion ist mit nur 120 Minuten insgesamt geplant, dadurch stellt BDD-NET eine ökonomisch günstige Intervention im Sinne der direkten Kosten dar. Als Nachteil aus diesem Setting ergibt sich, dass bei der Behandlung der KDS besonders auf den Faktor "Menschlichkeit zeigen" geachtet werden soll, z.B. durch das Eingestehen eigener Fehler und Schwierigkeiten [Brunhoeber, 2019]. Dieser Aspekt ist aufgrund des vergleichsweise geringeren therapeutischen Kontaktes nur schwer umzusetzen. Die bisherigen Evaluationen im englischen und schwedischen Sprachraum zeigen vielversprechende Symptomrückgänge [Enander et al., 2014, 2016; Gentile et al., 2019; Wilhelm et al., 2020]. Da jedoch die Gruppe der Betroffenen u.a. aufgrund hoher Komorbiditäten heterogen ist, kann erst die weitere wissenschaftliche Evaluation mit größer angelegten RCTs zeigen, ob sich die Effekte von BDD-NET weiter generalisieren lassen. Eine Vielzahl von Behandlungsbarrieren [Schulte et al., 2020] führt dazu, dass bisher nur ein kleiner Teil der Betroffenen eine adäquate Therapie erhält. Daher könnte ein internetgestütztes Programm ein erster Schritt sein, um z.B. die krankheitsimmanente Ambivalenz der Störungseinsicht abzubauen und somit einen anderen Behandlungsweg aufzuzeigen. Neben BDDNET prüfen aktuell laufende Studien auch die Eignung solcher internetgestützten Programme für andere Zielgruppen mit KDS, u.a. ImaginYouth für Jugendliche [Hartmann et al., 2021]. Es erstaunt nicht, dass BDDNET in England als Bestandteil einer Stepped-Care-Behandlung von der aktuellen Leitlinie empfohlen wird [NICE, 2019]. Dies kann möglicherweise im Rahmen eines Stepping-Up auch den Weg in die ambulante Psychotherapie ebnen.

Da das durchschnittliche Alter bei Störungsbeginn in der Jugend liegt [Gunstad und Phillips, 2003] und gerade für diese Altersgruppe E-Mental-Health-Angebote aufgrund einer höheren Technikaffinität attraktiv scheinen, ist die deutschsprachige Entwicklung einer internetgestützten Therapie folgerichtig. Das Setting des Internets/ Computers könnte sogar dem des Smartphones überlegen sein, da es mit einer Arbeitsplatzsituation assoziiert werden und somit zu einer günstigen Leseatmosphäre führen könnte. Demgegenüber ist zu beachten, dass das Internet einer Vielzahl von Betroffenen auch als Vergleichsmöglichkeit (z.B. Social Media) dient. Zudem bergen Bildschirme die Möglichkeit der Spiegelung. Beides könnte negativ assoziiert sein, was die Therapiemotivation senken könnte.

Die ausgeprägte Scham kann für KDS-Betroffene eine Behandlungsbarriere darstellen. Möglicherweise kann hier ein E-Mental-Health-Programm als leichter zugänglich wirken als der Gang in eine psychotherapeutische Praxis. Auch könnten stark schambesetzte Themen durch die räumliche Distanz möglicherweise einfacher angesprochen werden. Gleichzeitig könnte das Programm jedoch auch dazu einladen, weiterhin in der "Vermeidung" zu bleiben und soziale Isolation fördern. Der Gang zu einer psychotherapeutischen Praxis als möglicher erster Schritt aus dieser Vermeidung heraus würde wegfallen. Neben all diesen Einschränkungen zeigt sich die Studienlage als vielversprechend, dass EMental-Health-Angebote die KDS-Symptomatik signifikant und längerfristig senken können [Enander et al., 2014, 2016, 2019; Gentile et al., 2019]. Insgesamt könnte BDD-NET dazu beitragen, das Behandlungsangebot zu flexibilisieren und mehr Personen zu erreichen sowie eine kosteneffektive Behandlungsmöglichkeit zu etablieren. Eine Erfolgskontrolle auch mit Hinzunahme von langfristigem Follow-Up sowie differentiellen Prädiktoranalysen erscheint wichtig, um diejenigen Patienten $\mathrm{zu}$ identifizieren, die intensivere Behandlung im Sinne eines Stepping-Ups benötigen.

\section{Schlussfolgerung}

Bisher gibt es keine Behandlung, die allen KDS-Betroffenen hilft. Die Behandlung der KDS kann zudem vor dem Hintergrund verschiedener Faktoren langwierig und schwer sein. Eine erfolgsversprechende Intervention als Ergänzung zur Face-to-Face Psychotherapie stellt in der englischen und schwedischen Sprache das Programm BDD-NET dar. Gerade, da Behandlungsbarrieren bei KDS-Betroffenen vergleichsweise hoch sind, nehmen weitere niedrigschwellige Behandlungsmodalitäten, wie z.B. Telefontherapie oder internetgestützte Therapien, einen wichtigen Stellenwert ein. Das Medium des Internets führt zu bestimmten therapeutischen Besonderheiten im Vergleich zur Face-to-Face-Psychotherapie - so können stark schambesetzte Themen durch die körperliche Distanz vielleicht eher aufgegriffen werden. Daher könnte BDD-NET sowie niedrigschwellige Behandlungsangebote generell bei entsprechender Einbindung in ein Gesamtversorgungskonzept (wie insbesondere im SteppedCare-Ansatz umgesetzt) ein wichtiges komplementäres Angebot in der Versorgung von Menschen mit KDS darstellen.

\section{Statement of Ethics}

Die Autorinnen bestätigen hiermit, dass für die vorliegende Arbeit kein Ethikvotum erforderlich ist.

\section{Conflict of Interest Statement}

Die Autorinnen erklären, dass keine Interessenkonflikte vorliegen. 


\section{Funding Sources}

Der Artikel wurde von keinen externen Geldgebern unterstützt.

Literatur

American Psychiatric Association. Diagnostic and statistical manual of mental disorders. 5th ed. 2013. https://doi.org/10.1176/appi. books.9780890425596.

Boldrini T, Schiano Lomoriello A, Del Corno F, Lingiardi V, Salcuni S. Psychotherapy during COVID-19: how the clinical practice of Italian psychotherapists changed during the pandemic. Front Psychol. 2020 Oct;11:591170.

Brunhoeber S. Kognitive Verhaltenstherapie bei Körperdysmorpher Störung: Ein Therapiemanual. Hogrefe Verlag; 2019. https://doi. org/10.1026/02859-000

Buhlmann U, Glaesmer H, Mewes R, Fama JM, Wilhelm S, Brähler E, et al. Updates on the prevalence of body dysmorphic disorder: a population-based survey. Psychiatry Res. 2010 Jun;178(1):171-5.

Buhlmann U. The German version of the Brown Assessment of Beliefs Scale (BABS): development and evaluation of its psychometric properties. Compr Psychiatry. 2014 Nov;55(8):1968-71.

Buhlmann U. Treatment barriers for individuals with body dysmorphic disorder: an internet survey.J Nerv MentDis.2011 Apr;199(4):26871.

Bundespsychotherapeutenkammer. Videobehandlungen - Eine Umfrage zu Erfahrungen von Psychotherapeut*innen [Internet]. 2020. Verfügbar unter: https://www.lpk-bw.de/ sites/default/files/news/2020/20201105bptk-studie-videobehandlung.pdf.

Castle D, Beilharz F, Phillips KA, Brakoulias V Drummond LM, Hollander E, et al. Body dysmorphic disorder: a treatment synthesis and consensus on behalf of the International College of Obsessive-Compulsive Spectrum Disorders and the Obsessive Compulsive and Related Disorders Network of the European College of Neuropsychopharmacology. Int Clin Psychopharmacol. 2021 Mar;36(2):6175 .

Didie ER, Menard W, Stern AP, Phillips KA. Occupational functioning and impairment in adults with body dysmorphic disorder. Compr Psychiatry. 2008 Nov-Dec;49(6):561-9.

Enander J, Andersson E, Mataix-Cols D, Lichtenstein L, Alström K, Andersson G, et al. Therapist guided internet based cognitive behavioural therapy for body dysmorphic disorder: single blind randomised controlled trial. BMJ. 2016 Feb;352:i241.

Enander J, Ivanov VZ, Andersson E, Mataix-Cols D, Ljótsson B, Rück C. Therapist-guided, Internet-based cognitive-behavioural therapy for body dysmorphic disorder (BDD-NET): a feasibility study. BMJ Open. 2014 Sep;4(9):e005923.

\section{Author Contributions}

M.D., U.B., F.A.D., A.J., J.H., O.D. und B.W: Konzeption und Umsetzung der Übersetzung von BDD-NET. C.R. und J.E: Konzeption und Umsetzung des Originalprogramms. Alle Autorinnen haben zur Erstellung des finalen Manuskripts beigetragen.
Enander J, Ljótsson B, Anderhell L, Runeborg M, Flygare O, Cottman O, et al. Long-term outcome of therapist-guided internet-based cognitive behavioural therapy for body dysmorphic disorder (BDD-NET): a naturalistic 2-year follow-up after a randomised controlled trial.BMJOpen.2019Jan;9(1):e024307.

Gentile AJ, La Lima C, Flygare O, Enander J, Wilhelm S, Mataix-Cols D, et al. Internet-based, therapist-guided, cognitive-behavioural therapy for body dysmorphic disorder with global eligibility for inclusion: an uncontrolled pilot study. BMJ Open. 2019 Mar;9(3):e024693.

Gunstad J, Phillips KA. Axis I comorbidity in body dysmorphic disorder. Compr Psychiatry. 2003 Jul-Aug;44(4):270-6.

Harrison A, Fernández de la Cruz L, Enander J, Radua J, Mataix-Cols D. Cognitive-behavioral therapy for body dysmorphic disorder: A systematic review and meta-analysis of randomized controlled trials. Clin Psychol Rev. 2016 Aug;48:43-51.

Hartmann AS, Grocholewski A, Buhlmann U. Körperdysmorphe Störung. Hogrefe Verlag; 2019. https://doi.org/10.1026/02669-000.

Hartmann AS, Schmidt M, Staufenbiel T, Ebert DD, Martin A, Schoenenberg K. ImaginYouth-A Therapist-Guided Internet-Based Cognitive-Behavioral Program for Adolescents and Young Adults With Body Dysmorphic Disorder: Study Protocol for a Two-Arm Randomized Controlled Trial. Front Psychiatry. 2021 May;12:682965.

Kelly MM, Brault ME, Didie ER. Psychosocial functioning and quality of life in body dysmorphic disorder. In: Phillips KA, editor. Body dysmorphic disorder: Advances in research and practice. Oxford: Oxford University Press; 2017. pp. 139-54.

Kroenke K, Spitzer RL. The PHQ-9: A new depression diagnostic and severity measure. Psychiatr Ann. 2002;32(9):509-15.

Marques L, Weingarden HM, Leblanc NJ, Wilhelm S. Treatment utilization and barriers to treatment engagement among people with body dysmorphic symptoms. J Psychosom Res. 2011 Mar;70(3):286-93.

Moessner M, Bauer S. E-Mental-Health und internetbasierte Psychotherapie: Auf dem Weg in die Versorgung. Psychotherapeut. 2017;62(3):251-66.

National Institute for Health and Care Excellence (NICE). Obsessive-compulsive disorder and body dysmorphic disorder: Treatment. Clinical guideline (CG31) [Internet]. 2005. Verfügbar unter: https://www.nice.org.uk/guidance $/ \operatorname{cg} 31$.
National Institute for Health and Care Excellence (NICE). BDD-NET for adults with body dysmorphic disorder [Internet]. 2019. Verfügbar unter: https://www.nice.org.uk/Media/Default/About/what-we-do/NICE-advice/ IAPT/iab-bdd-net-adults-body-dysmorphicdisorder-for-publication.pdf.

Phillips KA, Hollander E, Rasmussen SA, Aronowitz BR, DeCaria C, Goodman WK. A severity rating scale for body dysmorphic disorder: development, reliability, and validity of a modified version of the Yale-Brown Obsessive Compulsive Scale. Psychopharmacol Bull. 1997;33(1):17-22.

Phillips KA, Menard W, Fay C, Pagano ME. Psychosocial functioning and quality of life in body dysmorphic disorder. Compr Psychiatry. 2005 Jul-Aug;46(4):254-60.

Phillips KA, Menard W, Quinn E, Didie ER, Stout RL. A 4-year prospective observational follow-up study of course and predictors of course in body dysmorphic disorder. Psychol Med. 2013 May;43(5):1109-17.

Phillips KA, Menard W. Suicidality in body dysmorphic disorder: a prospective study. Am J Psychiatry. 2006 Jul;163(7):1280-2.

Phillips KA. The broken mirror: Understanding and treating body dysmorphic disorder. Oxford: Oxford University Press; 2005.

Schneider SC, Turner CM, Storch EA, Hudson JL. Body dysmorphic disorder symptoms and quality of life: the role of clinical and demographic variables. J Obsessive Compuls Relat Disord. 2019;21:1-5.

Schulte J, Schulz C, Wilhelm S, Buhlmann U. Treatment utilization and treatment barriers in individuals with body dysmorphic disorder. BMC Psychiatry. 2020 Feb;20(1):69.

Stangier U, Hungerbühler R, Meyer A, Wolter M. Diagnostische Erfassung der Körperdysmorphen Störung. Eine Pilotstudie. Nervenarzt. 2000 Nov;71(11):876-84.

Veale D, Eshkevari E, Kanakam N, Ellison N, Costa A, Werner T. The Appearance Anxiety Inventory: validation of a process measure in the treatment of body dysmorphic disorder. Behav Cogn Psychother. 2014 Sep;42(5):60516.

Veale D, Gledhill LJ, Christodoulou P, Hodsoll J. Body dysmorphic disorder in different settings: A systematic review and estimated weighted prevalence. Body Image. 2016 Sep;18:168-86.

Veale D, Neziroglu F. Body dysmorphic disorder: A treatment manual. Wiley-Blackwell; 2010. https://doi.org/10.1002/9780470684610.

Wilhelm S, Phillips KA, Steketee G. Cognitivebehavioral therapy for body dysmorphic disorder: A treatment manual. Guilford Press; 2013. 
Wilhelm S, Weingarden H, Greenberg JL, McCoy TH, Ladis I, Summers BJ, et al. Development and pilot testing of a cognitive-behavioral therapy digital service for body dysmorphic disorder. Behav Ther. 2020 Jan;51(1):15-26.

Wong MY, Gunasekeran DV, Nusinovici S, Sabanayagam C, Yeo KK, Cheng CY, et al. Telehealth Demand Trends During the CO-
VID-19 Pandemic in the Top 50 Most Affected Countries: infodemiological Evaluation. JMIR Public Health Surveill. 2021 Feb;7(2):e24445.

World Health Organization. International statistical classification of diseases and related health problems (10th ed) [Internet]. 2019. Verfügbar unter: https://icd.who.int/ browse10/2019/en.
World Health Organization. International statistical classification of diseases and related health problems (11th ed) [Internet]. 2020a. Verfügbar unter: https://icd.who.int/ en.

World Health Organization. The impact of COVID-19 on mental, neurological and substance use services: results of a rapid assessment. Geneva: WHO; 2020b. 